\title{
Production Smoothing in Developed Countries
}

\author{
Isamu Ginama ${ }^{*}$, Mitsuhiro Odaki ${ }^{2}$ \\ ${ }^{1}$ Hiroshima University of Economics, Hiroshima, Japan \\ ${ }^{2}$ Hiroshima University, Hiroshima, Japan \\ Email: ^ginama@hiroshima-u.ac.jp
}

How to cite this paper: Ginama, I. and Odaki, M. (2017) Production Smoothing in Developed Countries. Journal of Mathematical Finance, 7, 333-350. https://doi.org/10.4236/jmf.2017.72018

Received: March 24, 2017

Accepted: May 15, 2017

Published: May 19, 2017

Copyright $\odot 2017$ by authors and Scientific Research Publishing Inc. This work is licensed under the Creative Commons Attribution International License (CC BY 4.0).

http://creativecommons.org/licenses/by/4.0/

\section{(c) (i) Open Access}

\begin{abstract}
This paper extends the method of regressing production and sales variables on a set of seasonal dummy variables and a linear trend for empirical tests on the production smoothing hypothesis of inventory investment. Unit root testing procedures form the basis for constructing the bootstrap confidence intervals for the ratio of the variance of production to the variance of sales. Models used to test for a unit root when there are structural breaks in the linear time trend are converted to equations comprising deterministic and stochastic components. A limiting distribution of the test statistic is derived. Bootstrap resampling is conducted over deterministic parts of the converted equations to construct confidence intervals for the relative variance ratio for G7 countries. Primary findings for these countries are production-counter smoothing phenomena.
\end{abstract}

\section{Keywords}

Unit Root, Structural Breaks, Inventory Investment, BCa Confidence Intervals, Limiting Distribution

\section{Introduction}

This paper uses macroeconomic data from G7 countries related to production, sales, and inventory investment to test the production smoothing hypothesis of inventory investment of an earlier study [1]. The hypothesis holds that the stock of inventories serves as a buffer in response to demand shifts that have not been forecast. The analytical framework used for the present study is an extension of models reported in the relevant literature [2] [3], which include the tenets that industrial data from the United States (US) and Taiwan can be converted to a stationary series in the way that the industrial production and sales series are regressed on the seasonal dummy variables and the linear time trend. Those models estimate the production and sales variances as the sum of the variances of the 
seasonal dummy variables and the variance of the residuals. Wang [3] applied the bootstrap method to construct confidence intervals $(\mathrm{BCa})$ of the ratio of the variances of production and sales. Wang's method is adopted here. However, the stationarity of the residuals was not tested formally in studies reported in the literature [2] [3]. In applying their methodological framework to macroeconomic data (such as gross domestic product (GDP), inventory investment, and aggregate sales), it is crucially important to test whether these variables are trendstationary. Explicit unit root tests of these variables are expected to involve trend terms with or without structural breaks. In this paper, the unit root tests consider the possibility of endogenous structural break(s). The specified unit root test equations are converted to appropriate formulas on which bootstrap methodologies are applied to derive confidence intervals for the relative variances of production and sales. In so doing, the problem of whether inventory investment is stabilizing or destabilizing in business cycle phases is expected to be analyzed empirically on a better statistical basis.

Here and in the literature, G7 countries were chosen as the group of economically developed countries (Canada, France, Germany, the United Kingdom (UK), Italy, Japan, and the US), which were characterized by production countersmoothing [4] [5] [6] [7].

The paper is organized as follows. Section 2 presents descriptions of the analyzed data. Section 3 presents the unit root test models used for this study. Section 4 explains the econometric method used to construct the BCa bootstrap confidence intervals of the ratio of the variance of production to the variance of sales. Section 5 presents sketches of the proof that the presence of the deterministic seasonal dummy variables does not affect the limiting distribution for the test statistic in the equations to test for a unit root. The unit root test results for the production and sales of the G7 countries are presented. Section 6 presents and interprets the estimated $\mathrm{BCa}$ confidence intervals. Concluding remarks follow that section.

\section{Data}

Data used for this study were extracted primarily from the Datastream database (Thomson Inc.) ${ }^{1}$. Real quarterly time series for GDP and inventory investment, based on chain-linked price indices, were obtained for the US, the UK, and Canada. The US inventory investment, which is seasonally adjusted, is related only to the private sector. The Canadian GDP and inventory investment are also seasonally adjusted. The French and Italian price indices are expressed in terms of the fixed base method. France's inventory investment is seasonally adjusted.

${ }^{1}$ The database software Data stream is a global data platform that is used widely by universities and libraries such as Universiteit van Amsterdam, European University Institute, and Harvard Business School Baker Library. "It has historical, global coverage of equities, stock markets, commodities, and economic data. Information is updated daily." (Baker Library Home page). Data stream provides post-1980 macroeconomic data for Japan; these data are constructed on the System of National Accounts 1993 (1993 SNA) basis. However, the Reuters Ecowin database provides Japanese data over a longer period, using the 1968 SNA basis. The Japanese data in this paper are kept on a 1968 SNAbasis. 
The GDP deflator for Italy, which is not seasonally adjusted, is unavailable. Therefore, its producer price index, which is not seasonally adjusted, was used to deflate the Italian variables. The German nominal GDP and inventory investment, which are not seasonally adjusted, were deflated by the chain-linked GDP deflator, which is also not seasonally adjusted.

Seasonal variations are noteworthy when analyzing inventory fluctuations [2] [3] [8]. Data from Japan², the UK, Italy, and Germany are not seasonally adjusted. Seasonal variations are incorporated into the variation of production and sales for these countries. Data for Canada, France, and the US are seasonally adjusted for analyses in this paper because of the constraint on the availability of data that are not seasonally adjusted. Sales are defined as the GDP minus the inventory investment, as in the literature. GDP and sales are transformed to logarithmic terms before testing for a unit root.

One report of the relevant literature [9] defines some trend concepts for applying to time series data. It defines local trends as trends “...only for some finite time interval..." (Definition 9). This definition applies to the deterministic trends in the current paper.

The sample time periods differ slightly among related countries. The time periods analyzed in this study depend on whether significant results were obtainable for testing the variables for stationarity around the deterministic trend, with and without structural breaks.

For Germany, the first differences of production and sales were tested for a unit root in augmented Dickey-Fuller (ADF) equations [10] [11] [12] with seasonal dummy variables after attempting to analyze the possibility of trend stationary results for various sample periods on the levels of German data. German data proved to be trend-stationary, having no structural break in this specification. When referring to Germany in relation to production counter-smoothing, the descriptions are of West Germany. We extracted annual West German data from the International Monetary Fund's International Financial Statistics during 1957-1991 $1^{3}$.

Canadian data were partitioned into two non-consecutive sample time periods. The 13-year time interval between the two observation periods was not included in our analyses because the unit root test outcomes showed no significant results for the sample periods that included this interval.

\section{Method}

We use three models to test the production and sales time series for a unit root. Particular forms of the models presented in earlier reports [13] [14] are used for demonstration purposes.

(i) The ADF test with the linear time trend, that is

\footnotetext{
${ }^{2}$ The data deflator for Japan used here was constructed using the fixed base method.

${ }^{3}$ Attempts to construct consistent data for West Germany on a quarterly basis were unsuccessful because the inventory investment figures represented on different volumes of the dataset were incompatible.
} 


$$
\Delta y_{t}=\hat{\mu}+\hat{\beta} t+\hat{\alpha}_{1} y_{t-1}+\sum_{i=1}^{k} \hat{\beta}_{i} \Delta y_{t-i}+e_{t}
$$

where $\hat{\mu}, \hat{\beta}, \hat{\alpha}_{1}$ and $\hat{\beta}_{i}^{\prime}$ are the parameters and where $e_{t}$ is the residual. The value of $k$ is determined using the Akaike information criterion. Therein, $\Delta$ is the first difference operator.

(ii) The test of the model presented earlier [13] with a single endogenous structural break for the break type $\mathrm{C}$ specification is

$$
y_{t}=\hat{\mu}+\hat{\theta} D U_{t}+\hat{\beta} t+\hat{\gamma} D T_{t}+\hat{\alpha} y_{t-1}+\sum_{i=1}^{k} \hat{\beta}_{i} \Delta y_{t-i}+e_{t}
$$

where $\hat{\theta}$ and $\hat{\gamma}$ are parameters, and where the value of $k$ is determined as explained in earlier reports of the literature [13] [14] [15] $]^{4}$. Variables $D U_{t}$ and $D T_{t}$ are defined as described in the next paragraph.

Let the break point be denoted as $T_{B}$, then $D U_{t}=1$ for $t \geq T_{B}+1$, and $D U_{t}=0$ otherwise. $D T_{t}$ is defined such that $D T_{t}=t-T_{B}$ for $t \geq T_{B}+1$, and $D T_{t}=0$ otherwise.

(iii) The test of another model presented earlier [14] with two endogenous structural breaks for break type BB is

$$
y_{t}=\hat{\mu}+\hat{\theta} D T 1_{t}+\hat{\beta} t+\hat{\gamma} D T 2_{t}+\hat{\alpha} y_{t-1}+\sum_{i=1}^{k} \hat{\beta}_{i} \Delta y_{t-i}+e_{t}
$$

$D T j_{t}$ is defined such that $D T j_{t}=t-T_{B j+1}$ for $t \geq T_{B j}+1$, and $D T j_{t}=0$ otherwise, where $j=1,2$.

\section{Bootstrapping}

Following a procedure described in an earlier report [3], BCa bootstrap confidence intervals of the variance ratio, i.e., the ratio of the variance of production to the variance of sales, are constructed by bootstrapping the deterministic parts of Equations (1), (2), and (3). That study [3] relied upon the assumption that the production and sales time series are expressible as the sum of the deterministic terms comprising seasonal dummy variables and the linear time trend, and the stationary random residuals. However, another report of the literature [16] [17] presents conversion of Equations (1) - (3) for the unit root tests to the form specified in two earlier studies [2] [3].

(i) Converting Equation (1)

Letting $\hat{\alpha}=1+\hat{\alpha}_{1}$, then Equation (1) can be written as

$$
y_{t}=\hat{\mu}+\hat{\beta} t+\hat{\alpha} y_{t-1}+\sum_{i=1}^{k} \hat{\beta}_{i} \Delta y_{t-i}+e_{t},
$$

which can be abbreviated further to

$$
y_{t}=\hat{\mu}^{\prime}+\hat{\beta}^{\prime} t+O T_{t}^{\prime}
$$

where $\hat{\beta}^{\prime}=\hat{\beta}\left(\frac{1}{1-\hat{\alpha}}\right), \hat{\mu}=\frac{\hat{\mu}}{1-\hat{\alpha}}-\hat{\beta} \hat{\alpha}\left(\frac{1}{(1-\hat{\alpha})^{2}}\right)$, and

${ }^{4}$ The truncation value for the $\mathrm{t}$-statistic on $\hat{\beta}_{i}$ is 1.6 in absolute value. When the variables that are not seasonally adjusted are used in the ADF equations, this criterion is applied for the value of $k$. The maximum lag was eight, as reported by Zivot and Andrews [13], where annual data were used. Where quarterly data are used in this paper, the maximum value of $k$ is set as 12 . 
$O T_{t}^{\prime}=\frac{1}{1-\hat{\alpha} L}\left(\sum_{i=1}^{k} \hat{\beta}_{i} \Delta y_{t-i}+e_{t}\right)^{5}$. Using Equation (1)" for the GDP and the sales simultaneously, bootstrap resamplings on $y_{t}$ and $t$ are made to run a series of ordinary least squares (OLS) regressions with the bootstrap samples on equation (1)". The variance of the $O T_{t}^{\prime}$ term gives the variance of production for the GDP equation, and gives the variance of sales for the sales equation. Based on replications of the ratio of those variances, the $\mathrm{BCa}$ bootstrap confidence intervals can be constructed as explained in several reports of the relevant literature [18] [19] [20] [21].

(ii) Converting Equation (2)

In rewriting Equation (2), terms $\left(\frac{1}{1-\hat{\alpha} L}\right) D U_{t}$ and $\left(\frac{1}{1-\hat{\alpha} L}\right) D T_{t}$ must be defined. Let $D U C_{t}=\left(\frac{1}{1-\hat{\alpha} L}\right) D U_{t}$ and $D T C_{t}=\left(\frac{1}{1-\hat{\alpha} L}\right) D T_{t}$. Then it is straightforward to demonstrate that $D U C_{t}=\left(\frac{1-\hat{\alpha}^{i}}{1-\hat{\alpha}}\right)$ for $t=\left(T_{B}+i\right), i=1,2, \cdots, N-T_{B}$, and $D U C_{t}=0$ for $t<T_{B}$, and that $D T C_{\left(T_{B}+i\right)}=\sum_{j=1}^{i}\left[D T_{i}-(j-1)\right] \hat{\alpha}^{(j-1)}$ for $i=1,2, \cdots, N-T_{B}$, and $D T C_{t}=0$ for $t \leq T_{B}$. Here, $N$ stands for the number of observations. Using these terms, Equation (2) can be rewritten as

$$
y_{t}=\hat{\mu}^{\prime}+\hat{\theta} D U C_{t}+\hat{\beta}^{\prime} t+\hat{\gamma} D T C_{t}+O T_{t}^{\prime \prime}
$$

In $(2), \quad \hat{\mu}^{\prime}=\frac{\hat{\mu}}{1-\hat{\alpha}}-\hat{\beta} \hat{\alpha}\left(\frac{1}{(1-\hat{\alpha})^{2}}\right), \quad \hat{\beta}^{\prime}=\hat{\beta}\left(\frac{1}{1-\hat{\alpha}}\right)$, and

$O T_{t}^{\prime \prime}=\left(\frac{1}{1-\hat{\alpha} L}\right)\left(\sum_{i=1}^{k} \hat{\beta}_{i} \Delta y_{t-i}+e_{t}\right)$. Bootstrap resamplings are made with $y_{t}$, $D U C_{t}, t$, and $D T C_{t}$ to apply a series of OLS regressions with these variables on Equation (2)' for the GDP and sales series to calculate the variances of $O T_{t}^{\prime \prime}$. These give the variances of production and sales from which the $\mathrm{BCa}$ confidence intervals on the variance ratio are constructed.

(iii) Converting Equation (3)

Equation (3) can be written as

$$
y_{t}=\hat{\mu}^{\prime}+\hat{\theta} D T C 1_{t}+\hat{\beta}^{\prime} t+\hat{\gamma} D T C 2_{t}+O T_{t}^{\prime \prime} .
$$

Therein, $\quad D T C j_{\left(T_{B j}+i\right)}=\sum_{h=1}^{i}\left[D T j_{i}-(h-1)\right] \hat{\alpha}^{(h-1)} \quad$ for $\quad i=1,2, \cdots, N-T_{B}$, and $D T C j_{t}=0$ for $t \leq T_{B j}$, where $N$ stands for the number of observations, and where $j=1,2$.

(i) Seasonal dummy variables

Equation (3) with the seasonal dummy variables is written as

$$
y_{t}=\hat{\mu}+\varphi_{1} d 1+\varphi_{2} d 2+\varphi_{3} d 3+\hat{\theta} D T 1_{t}+\hat{\beta} t+\hat{\gamma} D T 2_{t}+\hat{\alpha} y_{t-1}+\sum_{i=1}^{k} \hat{\beta}_{i} \Delta y_{t-i}+e_{t} .
$$

That equation can be converted to

$$
y_{t}=\hat{\mu}^{\prime}+\varphi_{1} d c_{1}+\varphi_{2} d c_{2}+\varphi_{3} d c_{3}+\hat{\theta} D T C 1_{t}+\hat{\beta}^{\prime} t+\hat{\gamma} D T C 2_{t}+O T_{t}^{\prime \prime} .
$$

Table 1 presents definitions of $d c_{i}, i=1,2,3$. The variance of $y_{t}\left(V\left(y_{t}\right)\right)$

${ }^{5} L$ represents the lag operator. 
Table 1. Conversions of the seasonal dummy variables.

\begin{tabular}{rccc}
\hline & $d c 1_{t}$ & $d c 2_{t}$ & $d c 3_{t}$ \\
\hline $1^{\text {st }}$ quarter & $\frac{1}{1-\hat{\alpha}^{4}}$ & $\frac{\hat{\alpha}^{3}}{1-\hat{\alpha}^{4}}$ & $\frac{\hat{\alpha}^{2}}{1-\hat{\alpha}^{4}}$ \\
$2^{\text {nd }}$ quarter & $\frac{\hat{\alpha}}{1-\hat{\alpha}^{4}}$ & $\frac{1}{1-\hat{\alpha}^{4}}$ & $\frac{\hat{\alpha}^{3}}{1-\hat{\alpha}^{4}}$ \\
$3^{\text {rd }}$ quarter & $\frac{\hat{\alpha}^{2}}{1-\hat{\alpha}^{4}}$ & $\frac{\hat{\alpha}}{1-\hat{\alpha}^{4}}$ & $\frac{1}{1-\hat{\alpha}^{4}}$ \\
$4^{\text {th }}$ quarter & $\frac{\hat{\alpha}^{3}}{1-\hat{\alpha}^{4}}$ & $\frac{\hat{\alpha}^{2}}{1-\hat{\alpha}^{4}}$ & $\frac{\hat{\alpha}}{1-\hat{\alpha}^{4}}$ \\
\hline
\end{tabular}

Notes: The equation to test for a unit root of variable $y_{t}$ is $y_{t}=\hat{\mu}+\hat{\beta}_{1} d 1_{t}+\hat{\beta}_{2} d 2_{t}+\hat{\beta}_{3} d 3_{t}+\hat{\gamma} t+\hat{\alpha} y_{t-1}+e_{t}$, where $d i_{t}$ are the seasonal dummy variables that take a value of one in the th quarter $(i=1,2$, and 3$)$, and $t$, $e_{t}$ respectively denote the linear time trend and the residual. $\hat{\mu}, \hat{\beta}_{1}, \hat{\beta}_{2}, \hat{\beta}_{3}, \hat{\gamma}$, and $\hat{\alpha}$ are the parameters in the equation. The table presents values that the term $\frac{d i_{t}}{1-\hat{\alpha} L}=d c i_{t} \quad(I=1,2,3)$ takes in each quarter $(i=$ 1,2 , and 3) during observations.

in this equation is given as $V\left(y_{t}\right)=\frac{1}{4} \sum_{i=0}^{3}\left(\varphi_{i}-\bar{\varphi}\right)^{2}+V\left(O T_{t}^{\prime \prime}\right)$ as described in earlier reports [2] [3], where $\varphi_{0}=\hat{\mu}^{\prime}, \bar{\varphi}=\frac{1}{4} \sum_{i=0}^{3} \varphi_{i}$, and $V\left(O T_{t}^{\prime \prime}\right)$ stands for the variance of $O T_{t}^{\prime \prime}$.

Equations (1)', (2)', (3)', and (4)', or an appropriate combination of these equations, were applied to the production and the sales series. Bootstrap resamplings were conducted over the deterministic parts of the converted equations simultaneously, to construct the $\mathrm{BCa}$ confidence intervals for the relative variance ratio of production and sales.

\section{Unit Root Tests}

\subsection{Limiting Distribution}

One report of the relevant literature [22] derived a proposition by which limiting distribution for the test statistic in Equations (1) and (1) 'is unaffected by the presence of the deterministic seasonal dummy variables for the ADF test. We present a sketch of the proof of a similar proposition: that the inclusion of the deterministic seasonal dummy variables in the testing equation with a single endogenous break does not affect the limiting distribution of the test statistic. We surmise that the same proposition holds for other break types within the single break model. Furthermore, it holds for the nine break types within the two endogenous break models. See the Appendix for the proof.

\subsection{Estimation}

Table 2, Table 3(a), and Table 3(b) present the unit root test results on the production and the sales for G7 countries. In these tables, all the data are in real and logarithmic terms. The notation used for the break types follows that reported for other studies in the literature [13] [14] [15]. 
Table 2. Summary of the unit root tests.

\begin{tabular}{|c|c|c|c|c|c|c|c|c|}
\hline & \multicolumn{2}{|c|}{ Japan } & \multicolumn{2}{|c|}{ US } & \multicolumn{2}{|c|}{ France } & \multicolumn{2}{|c|}{ UK } \\
\hline & GDP & Sales & GDP & Sales & GDP & Sales & GDP & Sales \\
\hline Obs. & \multicolumn{2}{|c|}{ (1955.1-1999.1) } & \multicolumn{2}{|c|}{$(1967.1-2008.1)$} & \multicolumn{2}{|c|}{$(1978.1-008.1)$} & \multicolumn{2}{|c|}{$(1983.1-2008.1)$} \\
\hline \multicolumn{9}{|c|}{ Period I } \\
\hline \multicolumn{9}{|c|}{$(1967.1-1984.4)$} \\
\hline No. of Breaks & 2 & 2 & 2 & 2 & 0 & 1 & 1 & 1 \\
\hline \multicolumn{9}{|c|}{ Period II } \\
\hline \multicolumn{9}{|c|}{$(1985.1-2008.1)$} \\
\hline No.of Breaks & & & 2 & 2 & & & & \\
\hline \multicolumn{9}{|c|}{ Period I } \\
\hline Break Type & BB & BB & $\mathrm{AA}$ & $\mathrm{AA}$ & N/A & $\mathrm{C}$ & A & A \\
\hline \multicolumn{9}{|c|}{ Period II } \\
\hline & & & BB & BB & & & & \\
\hline \multicolumn{9}{|c|}{ Period I } \\
\hline TB1 & 1972.3 & 1972.3 & 1978.1 & 1978.1 & & 1995.2 & 1990.1 & 1990.4 \\
\hline TB2 & 1992.3 & 1992.3 & 1981.3 & 1981.3 & & & & \\
\hline \multicolumn{9}{|c|}{ Period II } \\
\hline TB1 & & & 1995.2 & 1995.2 & & & & \\
\hline TB2 & & & 2000.2 & 2000.2 & & & & \\
\hline \multicolumn{9}{|c|}{ Period I } \\
\hline $\begin{array}{c}\text { Level of } \\
\text { Significance }(\tau)\end{array}$ & ** & $* *$ & ** & * & * & * & $* *$ & $* *$ \\
\hline \multicolumn{9}{|c|}{ Period II } \\
\hline & & & * & $* * *$ & & & & \\
\hline Data type & NSA & NSA & SA & SA & SA & SA & NSA & NSA \\
\hline
\end{tabular}

Notes: Obs. and No. respectively stand for the sample period and number. $\tau$ denotes the $t$ ratio of $\hat{\alpha} .{ }^{*}$, ${ }^{* *}$, and ${ }^{* *}$ respectively denote $10 \%, 5 \%$, and $1 \%$ levels of significance. NSA and SA respectively stand for not seasonally adjusted and seasonally adjusted series for quarterly data. TBi stands for the $i$ th break point $(i=1,2)$.

We divided the US sample period into two, and applied these periods to the models to test for a unit root. In each time period, the US data were trend stationary with two structural breaks. The confidence intervals for the variance ratio were therefore constructed in each time period for the US. The results for Germany in Table 3(a) are those for the period after the reunification of West and East Germany in 1990. The GDP and the sales for this period were trendstationary in the first differences. Table 3(b) presents results for West Germany using annual data in first differences. Both the GDP and the sales are identified as trend stationary series in the ADF tests. Table $3(\mathrm{~b})$ presents the Canadian results for two observation periods. The BCa bootstrap confidence intervals for these results are presented hereinafter. 
Table 3. (a) Summary of the unit root tests. (b) Summary of unit root tests.

(a)

\begin{tabular}{ccccccc}
\hline & \multicolumn{2}{c}{ Italy } & \multicolumn{2}{c}{ Germany } & \multicolumn{2}{c}{ Canada } \\
\hline & GDP & Sales & $\Delta$ GDP & $\Delta$ Sales & GDP & Sales \\
\hline Obs. & $(1981.1-2007.1)$ & $(1991.1-2008.1)$ & $(1990.2-2008.1)$ \\
No. of Breaks & 2 & 2 & 0 & 0 & 2 & 2 \\
Break type & BC & BB & N/A & N/A & AB & CB \\
TB1 & 1991.3 & 1991.3 & & & 1998.2 & 1995.4 \\
TB2 & 2003.1 & 2003.3 & & & 2007.3 & 2002.1 \\
Level of Significance $(\tau)$ & $* *$ & $* *$ & $* * *$ & $* * *$ & $* * *$ & $* * *$ \\
Data type & NSA & NSA & NSA & NSA & SA & SA \\
\hline
\end{tabular}

(b)

\begin{tabular}{|c|c|c|c|c|c|c|}
\hline & \multicolumn{2}{|c|}{ Germany } & \multicolumn{2}{|c|}{ Canada } & \multicolumn{2}{|c|}{ Canada } \\
\hline & $\Delta \mathrm{GDP}$ & $\Delta$ Sales & GDP & Sales & GDP & Sales \\
\hline Obs. & \multicolumn{2}{|c|}{$(1957-1991)$} & \multicolumn{2}{|c|}{$(1965.1-1978.4)$} & \multicolumn{2}{|c|}{$(1992.1-2008.1)$} \\
\hline No. of Breaks & 0 & 0 & 0 & 0 & 1 & 1 \\
\hline Break type & $\mathrm{N} / \mathrm{A}$ & N/A & $\mathrm{N} / \mathrm{A}$ & $\mathrm{N} / \mathrm{A}$ & B & B \\
\hline TB1 & & & & & 2003.4 & 2004.2 \\
\hline \multicolumn{7}{|l|}{ TB2 } \\
\hline Level of Significance $(\tau)$ & $* * *$ & $* * *$ & * & * & ** & ** \\
\hline Data type & Annual & Annual & SA & SA & SA & SA \\
\hline
\end{tabular}

Notes: Obs. and No. respectively stand for the sample period and number. $\tau$ denotes the $t$ ratio of $\hat{\alpha} .{ }^{*}$, ${ }^{* *}$, and ${ }^{* *}$ respectively denote $10 \%, 5 \%$, and $1 \%$ levels of significance. NSA and SA respectively stand for not seasonally adjusted and seasonally adjusted series for quarterly data. TBi stands for the $i$ th break point $(i=1,2)$.

\section{BCa Bootstrap Confidence Intervals}

Tables 4-12 present the BCa bootstrap confidence intervals of the $90 \%, 95 \%$, and $99 \%$ confidence levels for G7 countries. In these tables, $V(y) / V(s), \quad \hat{z}_{0}$ and $\hat{a}$ respectively represent the ratio of the variance of production to the variance of sales, the bias correction constant, and the acceleration constant. $\hat{\theta}$ is the point estimate of the sample variance ratio. The number of bootstrap resampleings is 3,000 .

Japan, the US, France, and Italy show the production counter-smoothing property of inventory investment: The widest $99 \%$ confidence intervals represented are located in the region toward the right (greater) side of the value of one. Where the $90 \%$ confidence interval is applied, the UK is included in the group of countries for which production is more volatile than sales. Reports of the literature [4] [5] [6] [7] describing Germany as having production countersmoothing properties refer to West Germany during the period before German reunification. Table 3(b) presents West German unit root test results. The BCa confidence intervals calculated based on this model are presented in Table 10. 
Table 4. BCa confidence intervals for Japan (variance ratio).

\begin{tabular}{cc}
\hline \multicolumn{3}{c}{$V(y) / V(s)$} \\
\hline Confidence Interval & $\mathrm{BCa}$ \\
\hline $99 \%$ & $(1.01335,1.02716)$ \\
$95 \%$ & $(1.01473,1.02552)$ \\
$90 \%$ & $(1.01555,1.02448)$ \\
\hline
\end{tabular}

Notes: $\hat{z}_{0}=-0.00334, \hat{a}=0.04991$, and $\hat{\theta}=1.01993$. The sample period for the unit root test is 1955.1-1999.1 (no. observations $=177)$. The observation periods used to construct BCa confidence intervals were set as 1958.2-1999.1 (no. observations $=164)$.

Table 5. BCa confidence intervals for the United States (variance ratio).

\begin{tabular}{ccc}
\hline \multicolumn{3}{c}{$V(y) / V(s)$} \\
Confidence Interval & BCa \\
\hline Period & I & II \\
\hline $99 \%$ & $(1.18870,1.68141)$ & $(1.1120,1.5940)$ \\
$95 \%$ & $(1.25243,1.60796)$ & $(1.16848,1.52188)$ \\
$90 \%$ & $(1.28194,1.57434)$ & $(1.19710,1.48385)$ \\
\hline
\end{tabular}

Notes: $\hat{z}_{0}=-0.008356, \hat{a}=-0.002183$, and $\hat{\theta}=1.420776$ for period I, and $\hat{z}_{0}=-0.29324$, $\hat{a}=-0.008192$, and $\hat{\theta}=1.3542$ for period II. Periods I and II for the unit root tests were1967.1-1984.4 (no. observations $=72$ ) and 1985.1-2008.1 (no. observations = 93). Observation periods used to construct BCa confidence intervals for periods I and II were set respectively as1970.2-1984.4 and 1988.2-2008.1 (no. observations $=59$ and 80 ).

Table 6. BCa confidence intervals for France (variance ratio).

\begin{tabular}{cc}
\hline \multicolumn{2}{c}{$V(y) / V(s)$} \\
\hline Confidence Interval \\
$99 \%$ & $\mathrm{BCa}$ \\
$95 \%$ & $(1.084788,1.694079)$ \\
$90 \%$ & $(1.144478,1.597168)$ \\
\hline
\end{tabular}

Notes: $\hat{z}_{0}=-0.1916709, \hat{a}=0.012346076$, and $\hat{\theta}=1.364265449$. The sample period for the unit root test was 1978.1-2008.1 (no. observations $=121$ ). The observation period used to construct BCa confidence intervals was set as 1981.1-2008.1 (no. observations $=109$ ).

Table 7. BCa confidence intervals for United Kingdom (variance ratio).

\begin{tabular}{cc}
\hline & $\mathrm{V}(\mathrm{y}) / \mathrm{V}(\mathrm{s})$ \\
\hline Confidence Interval & $\mathrm{BCa}$ \\
\hline $99 \%$ & $(0.99879,1.00923)$ \\
$95 \%$ & $(0.99968,1.00733)$ \\
$90 \%$ & $(1.00011,1.00647)$ \\
\hline
\end{tabular}

Notes: $\hat{z}_{0}=0.06522, \hat{a}=0.04700$, and $\hat{\theta}=1.00261$. The sample period for the unit root test was 1983.1-2008.1 (no. observations $=101$ ). The observation period used to construct BCa confidence intervals was set as 1986.2-2008.1 (no. observations $=88$ ). 
Table 8. BCa confidence intervals for Italy (variance ratio).

\begin{tabular}{cc}
\hline \multicolumn{3}{c}{$V(y) / V(s)$} & \\
\hline Confidence Interval & BCa \\
\hline $99 \%$ & $(1.00023,1.00395)$ \\
$95 \%$ & $(1.00073,1.00361)$ \\
$90 \%$ & $(1.00097,1.00344)$ \\
\hline
\end{tabular}

Notes: $\hat{z}_{0}=0.05517, \hat{a}=-0.00131$, and $\hat{\theta}=1.00227$. The sample period for the unit root test was 1981.1-2007.1 (no. observations $=105$ ). The observation period used to construct BCa confidence intervals was set as 1984.2-2007.1 (no. observations $=92$ ).

Table 9. BCa confidence intervals for Germany (variance ratio).

\begin{tabular}{cc}
\hline \multicolumn{2}{c}{$V(y) / V(s)$} \\
\hline Confidence Interval \\
$99 \%$ & $(0.179115,0.289420)$ \\
$95 \%$ & $(0.189857,0.271006)$ \\
$90 \%$ & $(0.195748,0.262982)$ \\
\hline
\end{tabular}

Notes: $\hat{z}_{0}=0.021726, \hat{a}=0.018947$, and $\hat{\theta}=0.226948$. The sample period for the unit root test was 1991.1-2008.1 (no. observations $=69$ ). The observation period used to construct BCa confidence intervals was set as 1991.4-2008.1 (no. observations = 66).

Table 10. BCa confidence intervals for West Germany (variance ratio).

\begin{tabular}{cc}
\hline & $\mathrm{V}(\mathrm{y}) / \mathrm{V}(\mathrm{s})$ \\
\hline Confidence Interval & $\mathrm{BCa}$ \\
\hline $99 \%$ & $(1.006113,1.001788)$ \\
$95 \%$ & $(1.148905,1.852629)$ \\
$90 \%$ & $(1.233023,1.766839)$ \\
\hline
\end{tabular}

Notes: $\hat{z}_{0}=-0.090361, \hat{a}=-0.065289$, and $\hat{\theta}=1.522056$. The sample period for the unit root tests was 1957-1991 (no. observations $=35$ ). The observation period used to construct BCa confidence intervals was set as 1960-1991 (no. observations $=32$ ).

Table 11. BCa confidence intervals for Canada (variance ratio).

\begin{tabular}{cc}
\hline & \multicolumn{2}{c}{$V(y) / V(s)$} & \\
\hline Confidence Interval & BCa \\
\hline $99 \%$ & $(0.823454,1.843992)$ \\
$95 \%$ & $(0.910669,1.681195)$ \\
$90 \%$ & $(0.963495,1.592324)$ \\
\hline
\end{tabular}

Notes: $\hat{z}_{0}=-0.010027, \hat{a}=-0.041922$, and $\hat{\theta}=1.233036$. The sample period for the unit root test was 1965.1-1978.4 (no. observations $=56$ ). The observation period used to construct BCa confidence intervals was identical to the sample period. 
Table 12. BCa confidence intervals for Canada (variance ratio).

\begin{tabular}{cc}
\hline \multicolumn{2}{c}{$V(y) / V(s)$} \\
\hline Confidence Interval & BCa \\
\hline $99 \%$ & $(0.578803,1.333072)$ \\
$95 \%$ & $(0.666948,1.267492)$ \\
$90 \%$ & $(0.716487,1.204174)$ \\
\hline
\end{tabular}

Notes: $\hat{z}_{0}=0.274110, \hat{a}=0.002397$, and $\hat{\theta}=0.916256$. The sample period for the unit root test was set as 1992.1-2008.1 (no. observations $=65$ ). The observation period used to construct BCa confidence intervals was set as1995.2-2008.1 (no. observations $=52$ ).

From Table 10, production can be interpreted as being more volatile than sales, as described in the literature. However, the results for Germany presented in Table 9 show that production is smoother than sales. It might be the case that the reunification of West Germany and East Germany gave rise to a change in the relative volatilities of production and sales.

The probability that the calculation of the BCa bootstrap confidence intervals stops in the middle of the prescribed number of repetitions is not negligible when a break point of the time trend is located near the end of the sample period because the resampling process might then involve picking up only zeros for the dummy variable that represents a structural break. The second break point of the Canadian production series was found to be in the third period from the end of the sample period (Table 3(a)). The BCa bootstrap confidence intervals for Canada using this model are not reported because a series of OLS routines was suspended within the small number of repetitions. Because of this difficulty, the Canadian data were analyzed for two other observation periods. Results obtained for these cases are presented in Table 3(b). Using data from these time periods, Table 11 and Table 12 show that the estimated BCa bootstrap confidence intervals for Canada are located on both sides of the value of one, indicating that the test of the production-smoothing theory is indeterminate. The point estimate of the relative variance in Table 11 is greater than one. The relative variance of Canada documented in the literature [4] [5] [7] corresponds to this case. Interval estimates on the relative variance, however, are interpreted as inconclusive. The point estimate of the relative variance for the more recent sample period used in Table 12 took a value of less than 1. Results obtained from Canadian data differ from the implications of numerical estimates on the relative variances of production and sales in the literature.

\section{Concluding Remarks}

Point estimates of the relative variances of production and sales were used as the bases of the analyses in earlier reports of the relevant literature [4] [5] [7]. Methodologies represented in this paper extended one of hypothesis testing procedures used in earlier studies [2] [3] in the area of empirical examination of the production smoothing hypothesis of inventory investment. The unit root testing 
equations form the basis for constructing the BCa confidence intervals. Models used to test for a unit root, both with and without structural break(s) in a linear time trend, were converted to equations that comprise deterministic and stochastic components.

The confidence interval of the variance ratio enables interpretation of the test results of the production smoothing hypothesis of inventory investment, in terms of the relative volatility of production and sales in light of statistical variations of the sample data. A stylized fact [23] [24] [25] [26] supporting the hypothesis that production is more volatile than sales explains the inventory fluctuations of economically developed countries better than those of economically developing countries, according to an earlier paper [5]. Based on the interval estimation method presented in this paper, Japan, the US, France, the UK, and Italy apparently have production that is more volatile than sales.

Unlike results from the literature, results for Canada are indeterminate throughout the two time periods analyzed here. West German data show the production counter-smoothing property described in the literature. Germany since reunification, however, has less volatile production than sales.

It must be investigated whether the alleged international duality in the smoothing property of inventory investment remains as a remarkable feature in terms of the confidence interval estimations. Therefore, the analytical method presented in this paper should be implemented for other countries, particularly a group of economically developing countries.

When the sample period of a time series is sufficiently long to the degree that the number of break points exceeds two, then the number of combinations for the possible break types can render the computation burden implausible. The way to apply the methodologies represented in this paper in such occasions is to divide the sample period, so that each period constitutes a tractable dataset. This procedure, however, points to the shortcomings of the methods described in this paper in achieving consistent statistical analyses of the entire sample period of the data with a long time horizon. Another implicit disadvantage for the methods used for this study arises when a break point is located near the end of a sample period. Such was the case for the Canadian production time series in $\mathrm{Ta}$ ble 3(a).

\section{Acknowledgements}

Previous versions of this paper were presented at the Far East and South Asian Meeting of the Econometric Society (August 2009), and at the Singapore Economic Review Conference 2013 (August 2013). We are grateful to participants of those conferences for their helpful comments and discussions. Any remaining errors, however, are solely attributable to the authors.

\section{Funding}

The authors acknowledge financial support from the Japan Society for the Pro- 
motion of Science, Grant-in-Aid for Scientific Research (KAKENHI) (Grant Number 23530221).

\section{References}

[1] Holt, C., Modigliani, F., Muth, J. and Simon, H. (1960) Planning Production, Inventories, and Work Force. Prentice Hall, Englewood Cliffs.

[2] Krane, S.D. and Braun, S.N. (1991) Production Smoothing Evidence from Physical-Product Data. Journal of Political Economy, 99, 558-581.

https://doi.org/10.1086/261767

[3] Wang, H.J. (2002) Nominal Data and the Production Smoothing Hypothesis. Economics Letters, 76, 245-250.

[4] West, K.D. (1990) Evidence from Seven Countries on Whether Inventories Smooth Aggregate Output. Engineering Costs and Production Economics, 19, 85-90.

[5] Fukuda, S. and Teruyama, H. (1988) Some International Evidence on Inventory Fluctuations. Economics Letters, 28, 225-230.

[6] Wilkinson, M. (1989) Aggregate Inventory Behavior in Large European Economies. European Economic Review, 33, 181-194.

[7] Wilkinson, M. (1991) Inventory Behavior and Economic Instability in Japan. Journal of the Japanese and International Economies, 5, 189-198.

[8] Ghali, M.A. (1987) Seasonality, Aggregation and the Testing of the Production Smoothing Hypothesis. American Economic Review, 77, 464-469.

[9] White, H. and Granger, C. (2011) Consideration of Trends in Time Series. Journal of Time Series Econometrics, 3, 1-38. https://doi.org/10.2202/1941-1928.1092

[10] Dickey, D.A. and Fuller, W.A. (1979) Distribution of the Estimates for Autoregressive Time Series with a Unit Root. Journal of the American Statistical Association, 74, 427-431. https://doi.org/10.2307/2286348

[11] Dolado, J.J., Jenkinson, T. and Simon, S. (1990) Cointegration and Unit Roots. Journal of Economic Surveys, 4, 249-273. https://doi.org/10.1111/j.1467-6419.1990.tb00088.x

[12] MacKinnon, J.G. (1996) Numerical Distribution Functions for Unit Root and Cointegration Tests. Journal of Applied Econometrics, 11, 601-618. https://doi.org/10.1002/(SICI)1099-1255(199611)11:6<601::AID-JAE417>3.0.CO;2$\underline{\mathrm{T}}$

[13] Zivot, E. and Andrews, D.W.K. (1992) Further Evidence on the Great Crash, the Oil-Price Shock, and the Unit-Root Hypothesis. Journal of Business and Economic Statistics, 10, 251-270. https://doi.org/10.1080/07350015.1992.10509904

[14] Lumsdaine, R.L. and Papell, D.H. (1997) Multiple Trend Breaks and the Unit-Root Hypothesis. Review of Economics and Statistics, 79, 212-218. https://doi.org/10.1162/003465397556791

[15] Perron, P. (1989) The Great Crash, the Oil Price Shock, and the Unit Root Hypothesis. Econometrica, 57, 1361-1401. https://doi.org/10.2307/1913712

[16] Ginama, I., Odaki, M. and Wang, H.D. (2009) An International Study of Inventory Fluctuations. Discussion Paper Series, No. 2009-2, Faculty of Economics, Hiroshima University, Higashihiroshima.

[17] Wang, H.D. (2009) Stationarity of Chinese and International Macroeconomic Time Series, and the Test of the Production Smoothing Hypothesis. Doctorate Thesis, Graduate School of Social Sciences, Hiroshima University, Higashihiroshima. (In Japanese ) 
[18] Efron, B. (1981) Nonparametric Estimates of Standard Error: The Jackknife, the Bootstrap and Other Methods. Biometrika, 68, 589-599. https://doi.org/10.1093/biomet/68.3.589

[19] Efron, B. (1987) Better Bootstrap Confidence Intervals. Journal of the American Statistical Association, 82, 171-185. https://doi.org/10.1080/01621459.1987.10478410

[20] Efron, B. and Tibshirani, R.J. (1986) Bootstrap Methods for Standard Errors, Confidence Intervals, and Other Measure of Statistical Accuracy. Statistical Science, 1, 54-75. https://doi.org/10.1214/ss/1177013815

[21] Efron, B. and Tibshirani, R.J. (1998) An Introduction to the Bootstrap. Chapman \& Hall/CRC Press, London.

[22] Dickey, D.A., Bell, W.R. and Miller, R.B. (1986) Unit Roots in Time Series Models: Tests and Implications. American Statistician, 40, 12-26. https://doi.org/10.1080/00031305.1986.10475349

[23] Blanchard, O.J. (1983) The Production and Inventory Behavior of the American Automobile Industry. Journal of Political Economy, 91, 365-400. https://doi.org/10.1086/261154

[24] Blinder, A.S. (1986) Can the Production Smoothing Model of Inventory Behavior Be Saved? Quarterly Journal of Economics, 101, 431-454. https://doi.org/10.2307/1885691

[25] Kahn, J.A. (1987) Inventories and the Volatility of Production. American Economic Review, 77, 667-679.

[26] Ramey, V.A. (1991) Nonconvex Costs and the Behavior of Inventories. Journal of Political Economy, 99, 306-334. https://doi.org/10.1086/261752 


\section{Appendix}

Simply incorporating the ordinary dummy variables into Model (A) of [15] or fundamentally (A) of an earlier study [13], we obtain

$$
y_{t}=\mu_{1}+\left(\mu_{2}-\mu_{1}\right) D U_{t}+\theta t+\sum_{i=1}^{3} \gamma_{i} d_{i t}+\alpha y_{t-1}+\epsilon_{t}, \forall t \geq 1,
$$

where $D U_{t}$ follows the definition presented in an earlier report [13], as $D U_{t}=1$ if $t \geq T_{B}+1,0$ otherwise, $d_{i t}=1$ for any $t$ expressed as $t=4 \mathrm{~s}+i$ with a nonnegative integer $s, 0$ otherwise, where $i=1,2,3, y_{0}=O_{p}(1)$ or $O(1)$, and $\left\{\epsilon_{t}\right\}$ is a sequence of unobservable random variables that are iid with $E \epsilon_{t}=0, E \epsilon_{t}^{2}=\sigma^{2}>0$ and finite fourth-order and eighth-order cumulants. We also use the following regression equation

$$
\Delta y_{t}=\hat{\mu}_{1}+\left(\hat{\mu}_{2}-\hat{\mu}_{1}\right) D U_{t}+\hat{\theta} t+\sum_{i=1}^{3} \hat{\gamma}_{i} d_{i t}+(\hat{\alpha}-1) y_{t-1}+\hat{e}_{t}, t=2, \cdots, T,
$$

to obtain the test statistic, and in connection with Equation (5-2), let $l_{T}, l_{T_{B}}, \tau_{T}$ and $l_{i ; T}, i=1,2,3$ be the (T-1)-dimensional vectors composed respectively of 1 , $D U_{t}, t$ and $d_{i t}$, as

$$
l_{T}=(1, \cdots, 1)^{\prime}, \tau_{T}=(2,3, \cdots, T)^{\prime}, l_{T_{B}}=(0, \cdots, 0,1, \cdots, 1)^{\prime}
$$

provided that the $\left(T_{B}+1\right)$ th component is the first being 1 ,

$$
\begin{aligned}
& l_{1 ; T}=(1,0,0,0,1,0,0,0, \cdots)^{\prime}, \\
& l_{2 ; T}=(0,1,0,0,0,1,0,0,0, \cdots)^{\prime}, \\
& l_{3 ; T}=(0,0,1,0,0,0,1,0,0,0, \cdots)^{\prime},
\end{aligned}
$$

with breakpoint $T_{B}$ defined in the described above articles. Furthermore, let $\lambda=\lim _{T \rightarrow \infty} T_{B} / T$ and further define

$$
\begin{aligned}
& Y=\left(y_{2}, \cdots, y_{T}\right)^{\prime}, Y_{-1}=\left(y_{1}, \cdots, y_{T-1}\right)^{\prime}, \bar{E}=\left(\epsilon_{2}, \cdots, \epsilon_{T}\right)^{\prime}, \\
& \bar{S}=\left(\sum_{h=1}^{2} \epsilon_{h}, \sum_{h=1}^{3} \epsilon_{h}, \cdots, \sum_{h=1}^{T} \epsilon_{h}\right)^{\prime}, \bar{S}_{-1}=\left(\epsilon_{1}, \sum_{h=1}^{2} \epsilon_{h}, \cdots, \sum_{h=1}^{T-1} \epsilon_{h}\right)^{\prime}, \\
& X_{1}=\left[l_{T}, l_{T_{B}}, \tau_{T}\right], X_{2}=\left[l_{1 ; T}, l_{2 ; T}, l_{3 ; T}\right], \\
& X=\left[X_{1}, X_{2}\right], Z_{1}=\left[Y_{-1}, X_{1}\right], Z=\left[Y_{-1}, X\right], \\
& M_{1_{T}}=I_{T-1}-l_{T}\left(l_{T}^{\prime} l_{T}\right)^{-1} l_{T}^{\prime}, M_{X_{i}}=I_{T-1}-X_{i}\left(X_{i}^{\prime} X_{i}\right)^{-1} X_{i}^{\prime}, i=1,2, \\
& M_{X}=I_{T-1}-X\left(X^{\prime} X\right)^{-1} X^{\prime}, \\
& M_{Z_{1}}=I_{T-1}-Z_{1}\left(Z_{1}^{\prime} Z_{1}\right)^{-1} Z^{\prime}, M_{Z}=I_{T-1}-Z\left(Z^{\prime} Z\right)^{-1} Z^{\prime}, \\
& D_{T ; X}=\operatorname{diag}\left\{T^{1 / 2}, T^{1 / 2}, T^{3 / 2}, T^{1 / 2}, T^{1 / 2}, T^{1 / 2}\right\}, D_{T ; Z}=\left[\begin{array}{rr}
T & 0 \\
0 & D_{T ; X}
\end{array}\right] .
\end{aligned}
$$

Letting $T_{4}$ be the largest integer that is either equal to or less than $(T-1) / 4$ and noting that 


$$
\begin{aligned}
& \sum_{t=1}^{T_{4}}\{i+4(t-1)\}=i T_{4}+4 \sum_{t=1}^{T_{4}-1} t=i T_{4}+2\left(T_{4}-1\right)\left(T_{4}-2\right) \\
& =2 T_{4}^{2}+O(T)=T^{2} / 8+O(T), \quad i=1,2,3,
\end{aligned}
$$

then it is not difficult to confirm that

$$
D_{T ; X}^{-1} X^{\prime} X D_{T ; X}^{-1}=\left[\begin{array}{cccccc}
1 & 1-\lambda & \frac{1}{2} & \frac{1}{4} & \frac{1}{4} & \frac{1}{4} \\
1-\lambda & 1-\lambda & \frac{1-\lambda}{2} & \frac{1-\lambda}{4} & \frac{1-\lambda}{4} & \frac{1-\lambda}{4} \\
\frac{1}{2} & \frac{1-\lambda}{2} & \frac{1}{3} & \frac{1}{8} & \frac{1}{8} & \frac{1}{8} \\
\frac{1}{4} & \frac{1-\lambda}{4} & \frac{1}{8} & \frac{1}{4} & 0 & 0 \\
\frac{1}{4} & \frac{1-\lambda}{4} & \frac{1}{8} & 0 & \frac{1}{4} & 0 \\
\frac{1}{4} & \frac{1-\lambda}{4} & \frac{1}{8} & 0 & 0 & \frac{1}{4}
\end{array}\right]+O\left(T^{-1}\right)
$$

The test statistic $t_{\alpha}$ (i.e., the $t$-statistic of the regression coefficient $(\hat{\alpha}-1)$ in (5-2)) based on (5-2) is now expressed as

$$
t_{\alpha}(\lambda)=\left(T^{-1} Y^{\prime} M_{Z} Y\right)^{-\frac{1}{2}}\left(Y_{-1}^{\prime} M_{X} Y_{-1}\right)^{-\frac{1}{2}}\left\{Y_{-1}^{\prime} M_{X}\left(Y-Y_{-1}\right)\right\} .
$$

Furthermore, let $W_{A}(\lambda, r)$ denote a stochastic process on $[0,1]$, which is the projection residuals in $L_{2}[0,1]$ of a Brownian motion projected onto the subspace generated by 1 , du $(\lambda, r), r$ with du $(\lambda, r)$ such that du $(\lambda, r)=1$ if $r \geq \lambda$ and 0 otherwise, as done by Equations (9) and (10) in the literature [13]. Consequently, we have the following.

Lemma 1: Presume that $y_{t}$ is generated by (5-1). If the null is formulated as $\mu_{1}=\mu_{2}, \theta=\gamma_{1}=\gamma_{2}=\gamma_{3}=0$ and $\alpha=1$, and (5-2) is the regression for the unit root test, then the limiting distribution of $t_{\alpha}(\lambda)$ under the null is expressed as

$$
\left(\int_{0}^{1} W_{A}(\lambda, r)^{2} \mathrm{~d} r\right)^{-1 / 2}\left(\int_{0}^{1} W_{A}(\lambda, r) d W_{A}(\lambda, r)\right)
$$

Proof: First, for any integers $i, j$ such that $4>j>i>0$ and $s=1, \cdots, T_{4}$, $\sum_{n=i+1}^{j} \epsilon_{4(s-1)+n}$ is the sum of $(j-i)$ pieces of $\epsilon_{4(s-1)+n}$ and that $\sum_{n=i+1}^{j} \epsilon_{4(s-1)+n}$ and $\sum_{n=i+1}^{j} \epsilon_{4\left(s^{\prime}-1\right)+n}$ are independent as $s \neq s^{\prime}$. It is then shown by these matters that for $i, j$ given above

$$
\begin{aligned}
& T^{-3 / 2} \sum_{s=1}^{T_{4}} \sum_{h=1}^{4(s-1)+j} \epsilon_{h}-T^{-\frac{3}{2}} \sum_{s=1}^{T_{4}} \sum_{h=1}^{4(s-1)+i} \epsilon_{h} \\
& =T^{-3 / 2} \sum_{s=1}^{T_{4}}\left(\sum_{n=i+1}^{j} \epsilon_{4(s-1)+n}\right)=O_{P}\left(T^{-1}\right),
\end{aligned}
$$

which implies that

$$
T^{-3 / 2} \sum_{s=1}^{T_{4}} \sum_{h=1}^{4(s-1)+i} \epsilon_{h}=\left(\frac{1}{4}\right) T^{-3 / 2} \sum_{t=2}^{T} \sum_{h=1}^{t-1} \epsilon_{h}+O_{P}\left(T^{-1}\right), i=1,2,3,
$$

noting that 


$$
T^{-3 / 2} \sum_{t=2}^{T} \sum_{h=1}^{t-1} \epsilon_{h}=\sum_{i=1}^{3} T^{-3 / 2} \sum_{s=1}^{T_{4}} \sum_{h=1}^{4(s-1)+i} \epsilon_{h} .
$$

Next, note that the $(T-1)$ observations of Equation (5-1) under the null are written as

$$
Y=Y_{-1}+\bar{E}+\mu_{1} l_{T}=Y_{-1}+X b+\bar{E}=Z\left(1, b^{\prime}\right)^{\prime}+\bar{E},
$$

where $b$ is the coefficient vector defined suitably. This expression engenders

$$
t_{\alpha}(\lambda)=\left(T^{-1} \bar{E}^{\prime} M_{Z} \bar{E}\right)^{-1 / 2}\left(T^{-2} Y_{-1}^{\prime} M_{X} Y_{-1}\right)^{-\frac{1}{2}}\left(T^{-1} Y_{-1}^{\prime} M_{X} \bar{E}\right),
$$

noting that

$$
\begin{aligned}
T^{-2} Y_{-1}^{\prime} M_{X} Y_{-1}= & T^{-2} Y_{-1}^{\prime} M_{X_{1}} Y_{-1} \\
& -\left(T^{-\frac{3}{2}} Y_{-1}^{\prime} M_{X_{1}} X_{2}\right)\left(T^{-1} X_{2}^{\prime} M_{X_{1}} X_{2}\right)^{-1}\left(T^{-\frac{3}{2}} X_{2}^{\prime} M_{X_{1}} Y_{-1}\right), \\
T^{-1} Y_{-1}^{\prime} M_{X} \bar{E}= & T^{-1} Y_{-1}^{\prime} M_{X_{1}} \bar{E}-\left(T^{-\frac{3}{2}} Y_{-1}^{\prime} M_{X_{1}} X_{2}\right)\left(T^{-1} X_{2}^{\prime} M_{X_{1}} X_{2}\right)^{-1}\left(T^{-\frac{1}{2}} X_{2}^{\prime} M_{X_{1}} \bar{E}\right) .
\end{aligned}
$$

In view of Equation (5-3) and

$$
y_{t-1}=\sum_{h=1}^{t-1} \epsilon_{h}+(t-1) \mu_{1}+y_{0},
$$

because of Equation (5-1) under the null and noting that

$$
M_{X_{1}}=M_{1_{T}}-M_{1_{T}} \tau\left(\tau^{\prime} M_{1_{T}} \tau\right)^{-1} \tau^{\prime} M_{1_{T}},
$$

it is trivial to see that

$$
\left(T^{-1} X_{2}^{\prime} M_{X_{1}} X_{2}\right)^{-1}=O(1),
$$

and that

$$
\begin{aligned}
& T^{-\frac{3}{2}} Y_{-1}^{\prime} M_{X_{1}} X_{2}=T^{-\frac{3}{2}} \bar{S}_{-1}^{\prime} M_{X_{1}} X_{2}=T^{-\frac{3}{2}} \sum_{t=2}^{T} \bar{S}_{t-1}\left(d_{1 t}, d_{2 t}, d_{3 t}\right) \\
& -\left(T^{-\frac{3}{2}} \sum_{t=2}^{T} \bar{s}_{t-1}\right)\left\{(T-1)^{-1} \sum_{t=2}^{T}\left(d_{1 t}, d_{2 t}, d_{3 t}\right)\right\} \\
& -\left(T^{-\frac{5}{2}} \bar{S}_{-1}^{\prime} M_{1_{T}} \tau_{T}\right)\left(T^{-3} \tau_{T}^{\prime} M_{1_{T}} \tau_{T}\right)^{-1}\left[T^{-2} \sum_{t=2}^{T} t\left(d_{1 t}, d_{2 t}, d_{3 t}\right)\right. \\
& \left.-T^{-2}\left(\sum_{t=2}^{T} t\right)\left\{(T-1)^{-1} \sum_{t=2}^{T}\left(d_{1 t}, d_{2 t}, d_{3 t}\right)\right\}\right],
\end{aligned}
$$

where $\bar{s}_{t-1}=\sum_{h=1}^{t-1} \epsilon_{t}$. Because $\frac{T_{4}}{T}=\frac{1}{4}+O\left(T^{-1}\right)$ and $d_{i t}=1$ for any $t$ expressed as $t=4 s+i$ with a nonnegative integer $s, 0$ otherwise, by definition, we obtain

$$
\begin{aligned}
& T^{-3 / 2} \sum_{t=2}^{T} \bar{s}_{t-1}\left(d_{1 t}, d_{2 t}, d_{3 t}\right) \\
& =T^{-3 / 2} \sum_{s=1}^{T_{4}}\left(\sum_{h=1}^{4(s-1)+1} \epsilon_{h}, \sum_{h=1}^{4(s-1)+2} \epsilon_{h}, \sum_{h=1}^{4(s-1)+3} \epsilon_{h}\right)+O_{P}\left(T^{-1}\right), \\
& \left(T^{-3 / 2} \sum_{t=1}^{T} \bar{s}_{t-1}\right)\left\{(T-1)^{-1} \sum_{t=2}^{T}\left(d_{1 t}, d_{2 t}, d_{3 t}\right)\right\} \\
& =\left(T^{-3 / 2} \sum_{t=2}^{T} \sum_{h=1}^{t-1} \epsilon_{h}\right)\left(\frac{1}{4}, \frac{1}{4}, \frac{1}{4}\right)+O_{P}\left(T^{-1}\right),
\end{aligned}
$$




$$
\begin{aligned}
& T^{-2} \sum_{t=2}^{T} t\left(d_{1 t}, d_{2 t}, d_{3 t}\right)=\left(\frac{1}{8}, \frac{1}{8}, \frac{1}{8}\right)+O\left(T^{-1}\right) \\
& T^{-2}\left(\sum_{t=2}^{T} t\right)\left\{(T-1)^{-1} \sum_{t=2}^{T}\left(d_{1 t}, d_{2 t}, d_{3 t}\right)\right\}=\left(\frac{1}{8}, \frac{1}{8}, \frac{1}{8}\right)+O\left(T^{-1}\right) .
\end{aligned}
$$

Using Equation (A.6) as well as Equation (A.1), (A.5) is converted into

$$
T^{-3 / 2} Y_{-1}^{\prime} M_{X_{1}} X_{2}=O_{P}\left(T^{-1}\right) \text {. }
$$

It is then derived from (A.3), (A.4), and (A.7) that

$$
\begin{aligned}
& T^{-2} Y_{-1}^{\prime} M_{X} Y_{-1}=T^{-2} Y_{-1}^{\prime} M_{X_{1}} Y_{-1}+O_{P}\left(T^{-2}\right), \\
& T^{-1} Y_{-1}^{\prime} M_{X} \bar{E}=T^{-1} Y_{-1}^{\prime} M_{X_{1}} \bar{E}+O_{P}\left(T^{-1}\right) .
\end{aligned}
$$

It also follows from the well-known asymptotic results on the $\mathrm{I}(0)$ and $\mathrm{I}(1)$ series that

$$
\begin{aligned}
& T^{-1} \bar{E}^{\prime} M_{Z} \bar{E}=T^{-1} \bar{E}^{\prime} \bar{E}+O_{P}\left(T^{-1}\right)=\sigma^{2}+O_{P}\left(T^{-\frac{1}{2}}\right), \\
& T^{-1} \bar{E}^{\prime} M_{Z_{1}} \bar{E}=T^{-1} \bar{E}^{\prime} \bar{E}+O_{P}\left(T^{-1}\right)=\sigma^{2}+O_{P}\left(T^{-\frac{1}{2}}\right) .
\end{aligned}
$$

Putting Equations (A.2), (A.8), and (A.9) together shows that the limiting distribution of $t_{\alpha}(\lambda)$ is equal to that of

$$
\left(T^{-1} \bar{E}^{\prime} M_{Z_{1}} \bar{E}\right)^{-\frac{1}{2}}\left(T^{-2} Y_{-1}^{\prime} M_{X_{1}} Y_{-1}\right)^{-\frac{1}{2}}\left(T^{-1} Y_{-1}^{\prime} M_{X_{1}} \bar{E}\right) \text {. }
$$

One readily finds that this quantity is the same as that derived from the test statistic for Model (A) of earlier papers [13] [15], noting that the seasonal dummies are excluded from the list of the regressors in construction. Consequently, it is established by results or arguments in the articles described above that the limiting distribution of the quantity above is that claimed by the lemma.

Scientific Research Publishing

\section{Submit or recommend next manuscript to SCIRP and we will provide best} service for you:

Accepting pre-submission inquiries through Email, Facebook, LinkedIn, Twitter, etc. A wide selection of journals (inclusive of 9 subjects, more than 200 journals)

Providing 24-hour high-quality service

User-friendly online submission system

Fair and swift peer-review system

Efficient typesetting and proofreading procedure

Display of the result of downloads and visits, as well as the number of cited articles

Maximum dissemination of your research work

Submit your manuscript at: http://papersubmission.scirp.org/

Or contact jmf@scirp.org 